Article

\title{
On the Fibration Defined by the Field Lines of a Knotted Class of Electromagnetic Fields at a Particular Time
}

\author{
Manuel Arrayás ${ }^{+}$(D) and José L. Trueba *,t \\ Área de Electromagnetismo, Universidad Rey Juan Carlos, Calle Tulipán s/n, 28933 Móstoles (Madrid), Spain; \\ manuel.arrayas@urjc.es \\ * Correspondence: joseluis.trueba@urjc.es \\ + These authors contributed equally to this work.
}

Received: 27 August 2017; Accepted: 6 October 2017; Published: 9 October 2017

\begin{abstract}
A class of vacuum electromagnetic fields in which the field lines are knotted curves are reviewed. The class is obtained from two complex functions at a particular instant $t=0$ so they inherit the topological properties of red the level curves of these functions. We study the complete topological structure defined by the magnetic and electric field lines at $t=0$. This structure is not conserved in time in general, although it is possible to red find special cases in which the field lines are topologically equivalent for every value of $t$.
\end{abstract}

Keywords: electromagnetic knots; torus knots; maxwell equations in vacuum

\section{Introduction}

The application of topology to physics has lit up the understanding of some observed properties in physical systems. In classical electromagnetism, with the application of topology one can obtain electromagnetic fields with nontrivial properties [1]. Even using topological concepts one can get quantization rules for the electromagnetic helicity [2] and the electric charge [3].

It is possible to find electromagnetic fields in vacuum where the field lines are the level curves of two complex scalar fields $\phi(\mathbf{r}, t)$, and $\theta(\mathbf{r}, t)$, being maps from $S^{3}$ to $S^{2}$. A map $f: S^{3} \mapsto S^{2}$ can be classified by a Hopf index in homotopy classes, and that is related to the linking number of the one dimensional manifolds which are the preimages of two different regular points in $S^{2}$. So the field lines can be linked in those electromagnetic fields.

From the maps $\phi(\mathbf{r}, t)$ and $\theta(\mathbf{r}, t)$, the magnetic field $\mathbf{B}$ and an electric field $\mathbf{E}$ are obtained as

$$
\begin{aligned}
& \mathbf{B}(\mathbf{r}, t)=\frac{\sqrt{a}}{2 \pi i} \frac{\nabla \phi \times \nabla \bar{\phi}}{(1+\bar{\phi} \phi)^{2}}, \\
& \mathbf{E}(\mathbf{r}, t)=\frac{\sqrt{a} c}{2 \pi i} \frac{\nabla \bar{\theta} \times \nabla \theta}{(1+\bar{\theta} \theta)^{2}},
\end{aligned}
$$

where $\bar{f}$ denotes the complex conjugates of $f$ and $i$ the imaginary unit. The constant $a$ carries the physical dimensions and $c$ is the speed of light.

Moreover, the fields constructed from maps $\phi$ and $\theta(1)$ satisfy

$$
\begin{aligned}
\mathbf{B}(\mathbf{r}, t) & =\frac{\sqrt{a}}{2 \pi i c(1+\bar{\theta} \theta)^{2}}\left(\frac{\partial \bar{\theta}}{\partial t} \nabla \theta-\frac{\partial \theta}{\partial t} \nabla \bar{\theta}\right), \\
\mathbf{E}(\mathbf{r}, t) & =\frac{\sqrt{a}}{2 \pi i(1+\bar{\phi} \phi)^{2}}\left(\frac{\partial \bar{\phi}}{\partial t} \nabla \phi-\frac{\partial \phi}{\partial t} \nabla \bar{\phi}\right),
\end{aligned}
$$


for being a solution of Maxwell's equations in vacuum. The expressions (1) and (2) are the original Rañada recipe to construct electromagnetic fields in vacuum.

Well known solutions as the Coulomb field, the plane electromagnetic wave, the stationary electromagnetic wave, the electromagnetic field into a cavity or the magnetic field created by an infinite solenoid present no big problems when studied as solutions of Equations (1) and (2), and the complex maps $\phi(\mathbf{r}, t), \theta(\mathbf{r}, t)$ have been obtained for them in previous references [4-6]. Those solutions show how the model of electromagnetic in term of maps from $S^{3}$ to $S^{2}$ can be used to study topological quantization conditions which can be included in Maxwell's equations to explain topological aspects of some physical quantities: charge, energy or magnetic flux.

The interest for the study of explicit solutions of Equations (1) and (2) also comes from the fact that a physical quantity called the electromagnetic helicity [2], is the sum of the Hopf indices of the maps as is explained in some references including $[7,8]$. Thus it turns out that solutions in which the magnetic or the electric lines form links or knots, i. e. show nontrivial topology, can be related to the physical concept of helicity and photon content of the field.

The first of these nontrivial solutions was obtained using the Hopf fibration. That solution is known as the Hopf-Rañada electromagnetic knot [1] or hopfion and is related to the Robinson congruence [9-11]. The maps $\phi(\mathbf{r}, t), \theta(\mathbf{r}, t)$ for the Hopf-Rañada electromagnetic knot are [4]

$$
\begin{aligned}
\phi & =\frac{(A X-T Z)+i(A Y+T(A-1))}{(A Z+T X)+i(A(A-1)-T Y)}, \\
\theta & =\frac{(A Y+T(A-1))+i(A Z+T X)}{(A X-T Z)+i(A(A-1)-T Y)},
\end{aligned}
$$

where $A=\left(R^{2}-T^{2}+1\right) / 2, R^{2}=X^{2}+Y^{2}+Z^{2}$. We will use upper-case letters as dimensionless quantities. In this field, any pair of magnetic lines or electric lines is a link, with linking numbers equal to 1 . This electromagnetic knot has been extensively studied [8,12-16]. A few generalizations of the Hopf-Rañada solution have been found (see [17] and references therein for a review). For example, in [4] it was showed that electromagnetic fields proportional to the Hopf-Rañada solution satisfy Equations (1) and (2) only if the proportionality constant is an integer number, so the Hopf index is equal to $n^{2}$. Solutions with negative Hopf index $-n^{2}$ were also found, all of them satisfying exactly Equations (1) and (2).

In this work, we revise the construction of a class of solutions [18] which, in the general case, satisfy equations (1) and (2) but for a particular time, this is for $\mathbf{B}\left(\mathbf{r}, t_{0}\right)$ and $\mathbf{E}\left(\mathbf{r}, t_{0}\right)$. At $t_{0}$, by construction there are two complex scalar fields $\phi_{0}(\mathbf{r}), \theta_{0}(\mathbf{r})$ which determine linked torus knots as field lines. This class contains in particular the cases discussed in the previous paragraph, i. e. the Hopf-Rañada electromagnetic knots. From the scalars maps $\phi_{0}(\mathbf{r}), \theta_{0}(\mathbf{r})$ we will obtain the Euler potentials $[13,17]$ of the field at that instant. The Euler potentials define manifolds and the field lines belong to the tangent bundle of those surfaces. The intersection of two surfaces are the field lines as we will show. We will give the expressions for the fields at any time, and we will end with some conclusions and remarks on future work.

It remains an open question if it is possible to find $\phi$ and $\theta$ for all the time or at least to prove their existence, and whether the topology is preserved or not in time.

\section{Initial Conditions for the Fields}

In this section we concentrate on the initial conditions of the fields. We will choose our maps in such a way that the field lines form torus knots [18]. A torus knot is a closed curve lying on a torus surface. It rotates $n$ times around a circle contained in the interior of the torus while circling the torus $m$ times around its axis. If $(n, m)$ are coprime we get a knot, otherwise we get a torus link and in this case the number of components is equal to the greatest common divisor of $n$ and $m$.

Let us take the maps $\phi_{0}, \theta_{0}: S^{3} \rightarrow S^{2}$. As previously discussed, the preimage of any point in $S^{2}$ will be a closed curve in $S^{3}$ and the linking number of any pair pair of curves is given by the 
Hopf index. We will denote the Hopf index as $H\left(\phi_{0}\right)$ and $H\left(\theta_{0}\right)$ for the maps $\phi_{0}$ and $\theta_{0}$ respectively. Using Equation (1) we can find the associated fields at $t=0$ as

$$
\begin{aligned}
& \mathbf{B}(\mathbf{r}, 0)=\frac{\sqrt{a}}{2 \pi i} \frac{\nabla \phi_{0} \times \nabla \bar{\phi}_{0}}{\left(1+\bar{\phi}_{0} \phi_{0}\right)^{2}}, \\
& \mathbf{E}(\mathbf{r}, 0)=\frac{\sqrt{a} c}{2 \pi i} \frac{\nabla \bar{\theta}_{0} \times \nabla \theta_{0}}{\left(1+\bar{\theta}_{0} \theta_{0}\right)^{2}} .
\end{aligned}
$$

Note that using vector identities, the relations (5) and (6) satisfy $\nabla \cdot \mathbf{B}=0$ and $\nabla \cdot \mathbf{E}=0$, which are two of the Maxwell equations which the fields in vacuum must satisfy, and in particular at $t=0$.

The constant $a$ in the SI units is related to the intensity of the electromagnetic field. Also in the SI units, the coordinates $(x, y, z, t)$ and the dimensionless ones $(X, Y, Z, T)$, are related by $(X, Y, Z, T)=$ $(x, y, z, c t) / L_{0}$. As before $c$ is the speed of light and $L_{0}$ is a characteristic length which quantifies the size of the knot. Indeed $L_{0}$ can be identified with the mean radius of the energy density of the electromagnetic field [12]. We will also use the relation

$$
\frac{r^{2}}{L_{0}^{2}}=\frac{x^{2}+y^{2}+z^{2}}{L_{0}^{2}}=X^{2}+Y^{2}+Z^{2}=R^{2} .
$$

For our torus-knotted electromagnetic fields [18], we take the maps,

$$
\begin{gathered}
\phi_{0}=\frac{(X+i Y)^{(n)}}{\left(Z+i\left(R^{2}-1\right) / 2\right)^{(m)}}, \\
\theta_{0}=\frac{(Y+i Z)^{(l)}}{\left(X+i\left(R^{2}-1\right) / 2\right)^{(s)}} .
\end{gathered}
$$

The notation $\eta^{(i)}$ means to leave the modulus of $\eta$ invariant while the phase of $\eta$ is multiplied by an integer number $i$.

Any regular value of the maps $\phi_{0}$ or $\theta_{0}$, has a 1-dimensional preimage in $R^{3}$ which depending on the integers $n, m, l, s$ is a torus knot [19]. For the map $\phi_{0}$, the Hopf index of a pair of such preimages is $H\left(\phi_{0}\right)=n m$ and for the map $\theta_{0}$ is $H\left(\theta_{0}\right)=l s$. The field lines are then linked torus knots.

Using the electromagnetic vector potentials $\mathbf{A}$ and $\mathbf{C}$, so that $\mathbf{B}=\nabla \times \mathbf{A}$ and $\mathbf{E}=\nabla \times \mathbf{C}$, we can define the magnetic and electric helicities. Their initial values can be calculated using Equations (5) and (6) and are proportional to the Hopf indexes of the maps. Thus,

$$
\begin{array}{r}
h_{m}(t=0)=\frac{1}{2 c \mu_{0}} \int d^{3} r \mathbf{A} \cdot \mathbf{B}=\frac{a}{2 c \mu_{0}} H(\phi), \\
h_{e}(t=0)=\frac{\varepsilon_{0}}{2 c} \int d^{3} r \mathbf{C} \cdot \mathbf{E}=\frac{a}{2 c \mu_{0}} H(\theta) .
\end{array}
$$

Here $\varepsilon_{0}=1 /\left(c^{2} \mu_{0}\right)$ is the vacuum electric permittivity and helicities have dimensions of angular momentum.

\section{The Fibration of the Electromagnetic Fields at $t=0$}

Let us consider the problem of describing at $t=0$, the field lines given by Equations (5) and (6). We will present and make use of the Euler potentials (see [17] and references therein). The field lines have the property of being tangent to the field vectors at any point of space. The magnetic line passing through $\mathbf{r}_{0}$ at time $t_{0}$ is the integral curve $\mathbf{r}(\tau)$ that satisfies

$$
\begin{aligned}
\frac{d \mathbf{r}(\tau)}{d \tau} & =\mathbf{B}\left(\mathbf{r}(\tau), t_{0}\right), \\
\left.\mathbf{r}(\tau)\right|_{\tau=0} & =\mathbf{r}_{0} .
\end{aligned}
$$


Here, the curve is parametrized by $\tau$ which is related to the arc length of the curve $d s=\sqrt{(d x)^{2}+(d y)^{2}+(d z)^{2}}$ by $d \tau=d s / B$, being $B$ the modulus of the magnetic field.

The Euler potentials are defined as two real functions $\alpha_{1}(\mathbf{r}, t)$ and $\alpha_{2}(\mathbf{r}, t)$ such that

$$
\mathbf{B}=\nabla \alpha_{1} \times \nabla \alpha_{2}
$$

Euler potentials determine magnetic surfaces for a constant value. The intersection of two magnetic surfaces gives the corresponding field line. Thus $\alpha_{1}\left(\mathbf{r}, t_{0}\right)=k_{1}, \alpha_{2}\left(\mathbf{r}, t_{0}\right)=k_{2}$ determine a electric field line.

For the electric field in vacuum, since $\nabla \cdot \mathbf{E}=0$, we can define also Euler potentials as real function $\beta_{1} \in R$ and $\beta_{2} \in R$ so that

$$
\mathbf{E}=\nabla \beta_{2} \times \nabla \beta_{1}
$$

Again the intersections of the magnetic and electric surfaces give the corresponding magnetic and electric field lines. In particular, $\beta_{1}\left(\mathbf{r}, t_{0}\right)=k_{1}^{\prime}, \beta_{2}\left(\mathbf{r}, t_{0}\right)=k_{2}^{\prime}$ determines a electric field line.

The complex scalar fields $\phi_{0}$ and $\theta_{0}$ in Equations (5) and (6) define Euler potentials of the magnetic and the electric field, and by construction the intersection of the surfaces of constant value are knotted field lines at initial time $t=0$. Let us then obtain the Euler potentials. Since $\phi_{0}$ is a complex map, we take its modulus $\left|\phi_{0}\right|=\sqrt{\left(\Re\left(\phi_{0}\right)\right)^{2}+\left(\Im\left(\phi_{0}\right)\right)^{2}}$ and argument $\mathcal{A}_{\phi_{0}}=\arctan \left(\Im\left(\phi_{0}\right) / \Re\left(\phi_{0}\right)\right)$, so that

$$
\phi_{0}=\left|\phi_{0}\right| e^{i \mathcal{A}_{\phi_{0}}}
$$

Then, by (5),

$$
\mathbf{B}(\mathbf{r}, t=0)=\sqrt{a} \nabla\left(\frac{1}{1+\left|\phi_{0}\right|^{2}}\right) \times \nabla\left(\frac{\mathcal{A}_{\phi_{0}}}{2 \pi}\right) .
$$

Thus the Euler potentials are

$$
\begin{array}{r}
\alpha_{1}(\mathbf{r})=\frac{1}{1+\left|\phi_{0}\right|^{2}}, \\
\alpha_{2}(\mathbf{r})=\frac{\mathcal{A}_{\phi_{0}}}{2 \pi} .
\end{array}
$$

For the electric field, we again take the modulus of the complex field $\theta_{0}$ as $\left|\theta_{0}\right|=\sqrt{\left(\Re\left(\theta_{0}\right)\right)^{2}+\left(\Im\left(\theta_{0}\right)\right)^{2}}$ and its argument as $\mathcal{A}_{\theta_{0}}=\arctan \left(\Im\left(\theta_{0}\right) / \Re\left(\theta_{0}\right)\right)$. Substituting into Equation (6) turns out to be,

$$
\mathbf{E}(\mathbf{r}, t=0)=-c \sqrt{a} \nabla\left(\frac{1}{1+\left|\theta_{0}\right|^{2}}\right) \times \nabla\left(\frac{\mathcal{A}_{\theta_{0}}}{2 \pi}\right) .
$$

Then the Euler potentials of the electric field turn out to be

$$
\begin{array}{r}
\beta_{1}(\mathbf{r})=\frac{1}{1+\left|\theta_{0}\right|^{2}}, \\
\beta_{2}(\mathbf{r})=\frac{\mathcal{A}_{\theta_{0}}}{2 \pi} .
\end{array}
$$


Thus for the set of non-null knotted electromagnetic fields at $t=0$, given by the complex functions (8) and (9), the Euler potentials are

$$
\begin{aligned}
& \alpha_{1}(\mathbf{r})=\frac{4 Z^{2}+\left(R^{2}-1\right)^{2}}{\left(R^{2}+1\right)^{2}}, \\
& \alpha_{2}(\mathbf{r})=\frac{1}{2 \pi}\left[n \arctan \left(\frac{Y}{X}\right)-m \arctan \left(\frac{2 Z}{R^{2}-1}\right)\right], \\
& \beta_{1}(\mathbf{r})=\frac{4 X^{2}+\left(R^{2}-1\right)^{2}}{\left(R^{2}+1\right)^{2}}, \\
& \beta_{2}(\mathbf{r})=\frac{1}{2 \pi}\left[l \arctan \left(\frac{Z}{Y}\right)-s \arctan \left(\frac{2 X}{R^{2}-1}\right)\right] .
\end{aligned}
$$

As pointed earlier, the magnetic lines are then given by the equations $\alpha_{1}=k_{1}$ and $\alpha_{2}=k_{2}$, and analogously $\beta_{1}=k_{1}^{\prime}$ and $\beta_{2}=k_{2}^{\prime}$ give the electric field lines.

Consider first the magnetic surfaces. In Figure 1, we plot some surfaces $\alpha_{1}(\mathbf{r})=k_{1}$, where $0 \leq k_{1} \leq 1$. From (20) the surfaces are given by

$$
\left(1-k_{1}\right) R^{4}-2\left(1+k_{1}\right) R^{2}+4 Z^{2}+\left(1-k_{1}\right)=0 .
$$

The magnetic surfaces for $k_{1}=0$ and $k_{1}=1$ are singular. For $k_{1}=1$, it is the Z-axis, and for $k_{1}=0$ we have the curve $X^{2}+Y^{2}=1$, so the surfaces degenerate to magnetic lines. For $0<k_{1}<1$, the magnetic surfaces $\alpha_{1}(\mathbf{r})=k_{1}$ are nested tori, meaning that the magnetic lines (5) will lie on these tori.

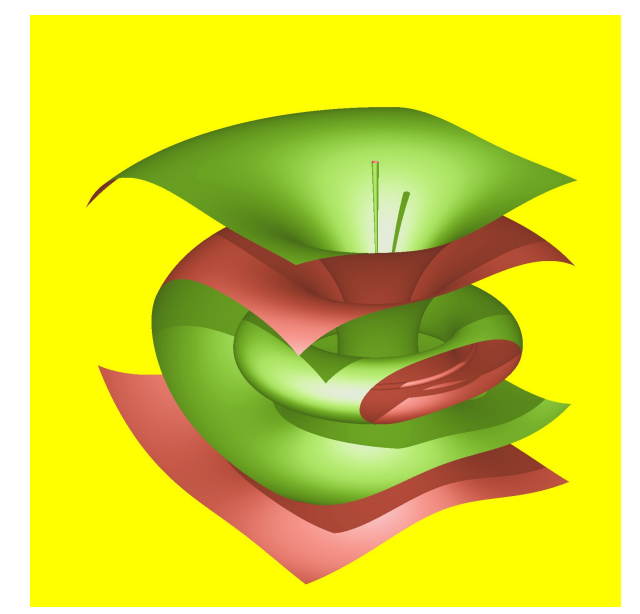

Figure 1. Magnetic surfaces given by the Euler potential $\alpha_{1}=k_{1}$, with $k_{1}=0.001,0.09,0.5,0.75,0.9998$.

The magnetic surfaces $\alpha_{2}(\mathbf{r})=k_{2}$ are given by

$$
n \arctan \left(\frac{Y}{X}\right)-m \arctan \left(\frac{2 Z}{R^{2}-1}\right)=2 \pi k_{2}
$$

for different values of $k_{2}$. The surfaces given by Equation (22) are not tori and we plot some particular cases in Figure 2. 


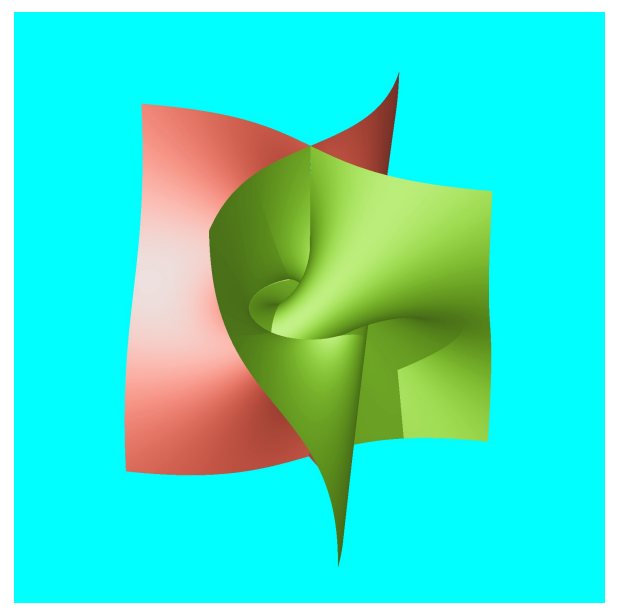

Figure 2. Magnetic surfaces given by the Euler potential $\alpha_{2}=k_{2}$, with $(n, m)=(1,1)$ and $k_{2}=0, \infty$.

The magnetic lines are the intersections of the surfaces $\alpha_{1}(\mathbf{r})=k_{1}$ with the surfaces $\alpha_{2}(\mathbf{r})=k_{2}$. In Figure 3 we have plotted the intersection of two magnetic surfaces. All these magnetic lines form linked torus knots.

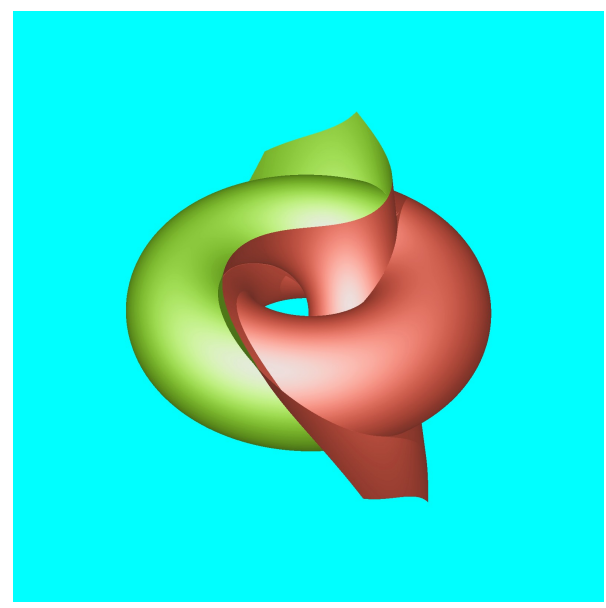

Figure 3. The intersection of magnetic surfaces for $\alpha_{1}=0.3$ and $\alpha_{2}=1 / 8$ for $(n, m)=(1,1)$.

The electric surfaces given by $\beta_{1}(\mathbf{r})=k_{1}^{\prime}$, with $0 \leq k_{1}^{\prime} \leq 1$, satisfy

$$
\left(1-k_{1}^{\prime}\right) R^{4}-2\left(1+k_{1}^{\prime}\right) R^{2}+4 X^{2}+\left(1-k_{1}^{\prime}\right)=0 .
$$

This equation is similar to (21) for the magnetic case, with the change $Z \rightarrow X$. Thus the electric surfaces $\beta_{1}(\mathbf{r})=k_{1}^{\prime}$ can be seen as a $\pi / 2$-rotation of the magnetic surfaces $\alpha_{1}(\mathbf{r})=k_{1}^{\prime}$ along the $Y$-axis. In Figure 4 we show some examples. Again the case $k_{1}^{\prime}=1$, is singular and the surface is the $X$-axis. For $k_{1}^{\prime}=0$ we have another singular surface, that is the circle $Y^{2}+Z^{2}=1$. For $0<k_{1}^{\prime}<1$, the electric surfaces $\beta_{1}(\mathbf{r})=k_{1}^{\prime}$ are nested tori. So the structure is the same as in Figure 1 with a $\pi / 2$ rotation around the $Y$-axis as we can see in Figure 4.

The electric surfaces $\beta_{2}(\mathbf{r})=k_{2}^{\prime}$ are given by

$$
l \arctan \left(\frac{Z}{Y}\right)-s \arctan \left(\frac{2 X}{R^{2}-1}\right)=2 \pi k_{2}^{\prime} .
$$


This expression can be obtained from (23) with the changes $X \rightarrow Y, Y \rightarrow Z, Z \rightarrow X, n \rightarrow l$, $m \rightarrow s$. As before, any electric field line, obtained as the intersection of a pair of electric surfaces, is topologically equivalent to a torus knot.

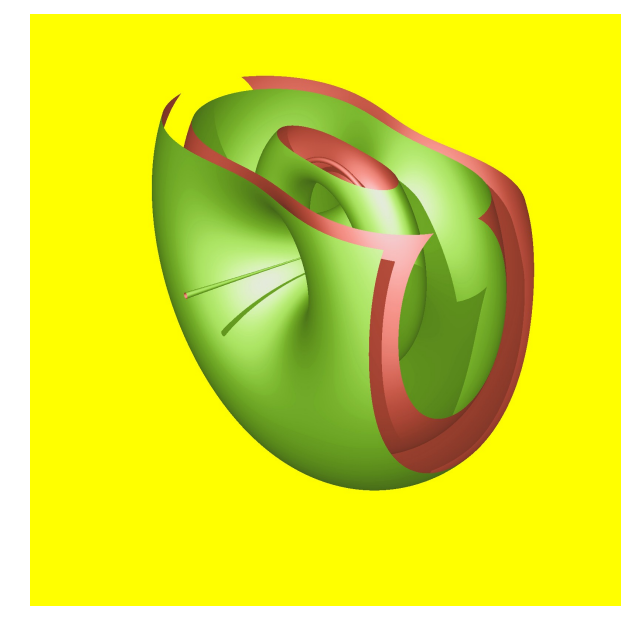

Figure 4. Magnetic surfaces given by the Euler potential $\beta_{1}=k_{1}^{\prime}$, with $k_{1}^{\prime}=0.001,0.09,0.5,0.6,0.9998$.

\section{The Vacuum Fields at Any Time}

Having discussed the initial configuration of the field lines of our torus knotted solutions, we can find the evolution of those field lines by solving the Cauchy problem for the system of Maxwell equations. Our initial configuration will be the initial conditions to satisfy. However, so far it is an open question if the magnetic and electric surfaces exist and can be explicitly calculated for the general case.

To solve Maxwell's equations in vacuum, Fourier analysis can be used. We give here the solutions obtained in [18]. Thus Maxwell fields in vacuum with the initial conditions given by expressions (5) and (6) are

$$
\begin{aligned}
& \mathbf{B}(\mathbf{r}, t)=\frac{\sqrt{a}}{\pi L_{0}^{2}} \frac{Q \mathbf{H}_{1}+P \mathbf{H}_{2}}{\left(A^{2}+T^{2}\right)^{3}}, \\
& \mathbf{E}(\mathbf{r}, t)=\frac{\sqrt{a} c}{\pi L_{0}^{2}} \frac{Q \mathbf{H}_{4}-P \mathbf{H}_{3}}{\left(A^{2}+T^{2}\right)^{3}},
\end{aligned}
$$

being

$$
\begin{aligned}
& A=\frac{R^{2}-T^{2}+1}{2}, \\
& P=T\left(T^{2}-3 A^{2}\right), \\
& Q=A\left(A^{2}-3 T^{2}\right),
\end{aligned}
$$


and

$$
\begin{array}{r}
\mathbf{H}_{1}=(-n X Z+m Y+s T) \mathbf{u}_{x}+(-n Y Z-m X-l T Z) \mathbf{u}_{y} \\
+\left(n \frac{-1-Z^{2}+X^{2}+Y^{2}+T^{2}}{2}+l T Y\right) \mathbf{u}_{z} \\
\mathbf{H}_{2}=\left(s \frac{1+X^{2}-Y^{2}-Z^{2}-T^{2}}{2}-m T Y\right) \mathbf{u}_{x} \\
+(s X Y-l Z+m T X) \mathbf{u}_{y}+(s X Z+l Y+n T) \mathbf{u}_{z} \\
\mathbf{H}_{3}=(-m X Z+n Y+l T) \mathbf{u}_{x}+(-m Y Z-n X-s T Z) \mathbf{u}_{y} \\
+\left(m \frac{-1-Z^{2}+X^{2}+Y^{2}+T^{2}}{2}+s T Y\right) \mathbf{u}_{z}, \\
\mathbf{H}_{4}=\left(l \frac{1+X^{2}-Y^{2}-Z^{2}-T^{2}}{2}-n T Y\right) \mathbf{u}_{x} \\
+(l X Y-s Z+n T X) \mathbf{u}_{y}+(l X Z+s Y+m T) \mathbf{u}_{z} .
\end{array}
$$

For the particular case $(n, m, s, l)=(1,1,1,1)$ we recover the Hopf-Rañada solution mentioned in the introduction section. For this particular case, maps $\phi$ and $\theta$ are known for any time, the Euler potentials can be found for any time, and the magnetic and electric helicities are constant. Using Equations (10) and (11) we can get the values of the Hopf index for any time, and prove that the topology property of the linking of curves is preserved in time. So the field lines remain closed and linked. This result can be generalized for the case $(n, m, l, s)=(1,1,1,1) \times N$, being $N$ an integer.

In the general case, for an arbitrary $(n, m, s, l)$-tuplet it remains an open question if the topology of the field lines is preserved. We have found numerical evidence of the existence of open curves for $T>0$.

\section{Discussion}

In this work, the set of non-null torus-knotted electromagnetic fields, presented in [18], have been reviewed. The fields have the property that at a given instant of time $t=0$, every magnetic line is a $(n, m)$-torus knot and is linked to another magnetic line. The electric lines are also $(l, s)$ torus knots, each one linked to any other electric line.

We have calculated the Euler potentials $[13,17]$ of such fields, and used them to study the configuration of electric and magnetic field lines at $t=0$. We have seen how the fibration defined by the Euler potentials at $t=0$ consists of linked torus knots.

It seems that the topological structure of the field lines is not preserved when the fields evolve in time in the general case, although it is preserved in the particular cases in which $n=m=l=s=N$ with $N$ an arbitrary integer. The complete understanding of the processes that produce the change of topology in some of these electromagnetic fields is an open problem.

Acknowledgments: We thank J. M. Montesinos Amilibia for useful discussions on Topology and Knot Theory, and to Á. Montesinos-Amilibia for his program Superficies (http://www.uv.es/montesin), which we have used to plot the figures. This work is supported by research grants from the Spanish Ministry of Economy and Competitiveness (MINECO/FEDER) ESP2015-69909-C5-4-R.

Author Contributions: Manuel Arrayás and José L. Trueba conceived all the results of this work, made the computations and wrote the paper.

Conflicts of Interest: The authors declare no conflict of interest. The founding sponsors had no role in the design of the study; in the collection, analyses, or interpretation of data; in the writing of the manuscript, and in the decision to publish the results. 


\section{References}

1. Rañada, A.F. A topological theory of the electromagnetic field. Lett. Math. Phys. 1989, 18, 97-106.

2. Trueba, J.L.; Rañada, A.F. The electromagnetic helicity. Eur. J. Phys. 1996, 17, 141.

3. Rañada, A.F.; Trueba, J.L. A topological mechanism of discretization for the electric charge. Phys. Lett. B 1998, 422, 196-200.

4. Rañada, A.F.; Trueba, J.L. Two properties of electromagnetic knots. Phys. Lett. A 1997, 232, 25-33.

5. Rañada, A.F. Interplay of topology and quantization: Topological energy quantization in a cavity. Phys. Lett. A 2003, 310, 434-444.

6. Rañada, A.F.; Trueba, J.L. Topological quantization of the magnetic flux. Found. Phys. 2006, 36, 427-463.

7. Arrayás, M.; Trueba, J.L. Exchange of helicity in a knotted electromagnetic field. Ann. Phys. 2012, 524, 71-75.

8. Rañada, A.F.; Trueba, J.L. Topological electromagnetism with hidden nonlinearity. In Modern Nonlinear Optics, Part 3; Evans, M.W., Ed.; John Wiley \& Sons, Inc.: New York, NY, USA, 2001; pp. 197-253.

9. Besieris, I.M.; Shaarawi, A.M. Hopf-Rañada linked and knotted light beam solution viewed as a null electromagnetic field. Opt. Lett. 2009, 34, 3887.

10. Robinson, I. Null electromagnetic fields. J. Math. Phys. 1961, 2, $290-291$.

11. Trautman, A. Solutions of the Maxwell and Yang-Mills equations associated with Hopf fibrings. Int. J. Theor. Phys. 1977, 16, 561-565.

12. Arrayás, M.; Trueba, J.L. Motion of charged particles in a knotted electromagnetic field. J. Phys A: Math. Theor. 2010, 43, 235401.

13. Van Enk, S.J. The covariant description of electric and magnetic field lines of null fields: Application to Hopf-Rañada solutions. J. Phys. A: Math. Theor. 2013, 46, 175204.

14. Irvine, W.T.M.; Bouwmeester, D. Linked and knotted beams of light. Nature Phys. 2008, 4, 716-720.

15. Rañada, A.F. Topological electromagnetism. J. Phys. A: Math. Gen. 1992, 25, 1621.

16. Rañada, A.F.; Trueba, J.L. Electromagnetic knots. Phys. Lett. A 1995, 202, 337-342.

17. Arrayás, M.; Bouwmeester, D.; Trueba, J.L. Knots in electromagnetism. Phys. Rep. 2017, 667, 1-61.

18. Arrayás, M.; Trueba, J.L. A class of non-null toroidal electromagnetic fields and its relation to the model of electromagnetic knots. J. Phys. A: Math. Theor. 2015, 48, 025203.

19. Dufraine, E. About homotopy classes of non-singular vector fields on the three-sphere. Qual. Theory Dyn. Syst. 2002, 3, 361-376. 